\title{
Oxygenation of (3Z)-Alkenals to 4-Hydroxy-(2E)-Alkenals in Plant Extracts: A Nonenzymatic Process
}

\author{
Minke A. Noordermeer,* I vo Feussner,†† Adelheid Kolbe, $\dagger$ \\ Gerrit A. Veldink,*,1 and J ohannes F.G. Vliegenthart* \\ *Bijvoet Center for Biomol ecular Research, Department of Bio-organic Chemistry, Utrecht U niversity, The Netherlands; \\ †I nstitute of Plant Biochemistry, Hall $\notin$ Saale, Germany; and łI nstitute of Plant Genetics and \\ Crop Plant Research, Gatersl eben, Germany
}

There is large interest in 4-hydroxy-(2E )-alkenals because of their cytotoxicity in mammals. However, the biosynthetic pathway for these compounds has not been elucidated yet. In plants, 4-hydroxy-(2E )-alkenals were supposed to be derived by the subsequent actions of lipoxygenase and a peroxygenase on (3Z)alkenals. The presence of 9-hydroxy-12-oxo-(10E)dodecenoic acid (9-hydroxy-traumatin) in incubations of 12-oxo-(9Z)-dodecenoic acid (traumatin) in the absence of lipoxygenase or peroxygenase, has prompted us to reinvestigate its mode of formation. We show here that in vitro 9-hydroxy-traumatin, 4-hydroxy(2E)-hexenal and 4-hydroxy-(2E)-nonenal, are formed in a nonenzymatic process. Furthermore, a novel product derived from traumatin was observed and identified as 11-hydroxy-12-oxo-(9Z)-dodecenoic acid. The results obtained here strongly suggest that the 4-hydroxy-(2E)-alkenals, observed in crude extracts of plants, are mainly due to autoxidation of (3Z)-hexenal, (3Z)-nonenal and traumatin. This may have implications for the in vivo existence and previously proposed physiological significance of these products in plants. $\odot 2000$ Academic Press

Key Words: 4-hydroxy-(2E)-alkenals; lipoxygenase pathway; 9-hydroxy-traumatin.

The formation of 4-hydroxy-(2E)-alkenals from polyunsaturated fatty acids was first observed in mammalian systems (1). Although these compounds have been

Abbreviations used: DNPH, 2,4-dinitrophenylhydrazone; FID, flame ionization detection; HHE, 4-hydroxy-(2E)-hexenal; HNE, 4-hydroxy-(2E )-nonenal; 13-HPOD, 13S-hydroperoxy-(9Z,11E)octadecadienoic acid; 13-HPOT, 13S-hydroperoxy-(9Z,11E,15Z)octadecatrienoic acid; HPO Iyase, hydroperoxide lyase; LOX, lipoxygenase.

${ }^{1}$ To whom correspondence should be addressed at Padualaan 8, $3584 \mathrm{CH}$ Utrecht, The Netherlands. Fax: +31 30 2540980. E-mail: veldink@accu.uu.nl. of great interest because of their cytotoxicity (2), the bi osynthetic pathway in mammals has not been unraveled yet. However, it has been suggested that at least 4-hydroxy-(2E)-nonenal (HNE) is formed by autoxidation (3).

In plant extracts, 4-hydroxy-(2E)-alkenals, such as HNE, 4-hydroxy-(2E)-hexenal (HHE) and 9-hydroxy12-oxo-(10E)-dodecenoic acid (9-hydroxy-traumatin), have been found as well (4-7). These compounds are suggested to be derived from (3Z)-hexenal, (3Z)nonenal and 12-oxo-(9Z)-dodecenoic acid (traumatin), which are products of the successive actions of lipoxygenase (LOX) and hydroperoxide lyase (HPO lyase) on linoleic and linolenic acid. Gardner and Hamberg proposed two enzymatic routes for the formation of these 4-hydroxy-(2E)-alkenals from (3Z)-alkenals in plants (8). First through an oxygenation reaction of a (3Z)alkenal to a 4-hydroperoxy-(2E )-alkenal by a (3Z)alkenal oxygenase activity, followed by reduction of the hydroperoxy group by a hydroperoxide-dependent peroxygenase. By investigating this process in more detail, it was found that the formation of 4-hydroperoxy(2E)-alkenals might be catalyzed by $\operatorname{LOX}(5,9)$. Alternatively, it was suggested that a 4-hydroxy-(2E)alkenal was produced by rearrangement of a 3,4epoxyalkenal which was also formed from a (3Z)alkenal by a hydroperoxide-dependent peroxygenase.

However, the finding of 9-hydroxy-traumatin in incubations of hydroperoxy fatty acids with pure HPO lyase, in the absence of LOX or a peroxygenase, has prompted us to reinvestigate its mode of formation (10, 11). The data we present here, indicate that the formation of 4-hydroxy-(2E)-alkenals from (3Z)-alkenals in vitro is mainly due to autoxidation.

\section{MATERIALS AND METHODS}

Enzyme preparations. Lipoxygenase-1 (LOX-1) was isolated from soybeans (12). Recombinant LOX-100 was isolated as described before (13). It is a 13-LOX which is induced by methyljasmonate and is 
located in chloroplasts of barley leaves (14). Recombinant HPO lyase was isolated as described previously (10). This HPO Iyase from alfalfa is highly specific for 13-hydroperoxy linoleic and linolenic acids. A crude extract of alfalfa was prepared as described previously (7).

Preparation of (3Z)-alkenals. (3Z)-hexenal and (3Z)-nonenal were prepared from the corresponding alcohols (3Z)-hexen-1-ol and (3Z)-nonen-1-ol, as described previously (15). 13S-hydroperoxy(9Z,11E,15Z)-octadecatrienoic acid (13-HPOT) and 13S-hydroperoxy-(9Z,11E)- octadecadienoic acid (13-HPOD) were prepared by incubation of $\alpha$-linolenic or linoleic acid with lipoxygenase-1 (16). Traumatin was prepared by incubation of HPO lyase with $100 \mu \mathrm{M}$ 13-HPOT in $50 \mathrm{mM}$ potassium phosphate buffer, $\mathrm{pH}$ 6.0. The reaction was followed by measuring the absorbance at $234 \mathrm{~nm}$ until all substrate was converted. The reaction mixture was acidified with $\mathrm{HCl}$ to $\mathrm{pH} 5.0$ and the traumatin was extracted with an octadecyl solid-phase extraction column (J . T. Baker B.V., Deventer, The Netherlands), previously equilibrated with $100 \%$ methanol. The column was washed with $50 \%$ methanol, and pure traumatin was obtained by elution with $75 \%$ methanol.

Formation of 4-hydroxy-(2E)-alkenals. For the formation of 9-hydroxy-traumatin, traumatin was incubated in a stirred vessel for $1.5 \mathrm{~h}$, in $5 \mathrm{ml}$ of $0.1 \mathrm{M}$ sodium borate buffer $\mathrm{pH} 9.0,50 \mathrm{mM}$ potassium phosphate buffer pH 6.0 or $50 \mathrm{mM}$ sodium acetate buffer $\mathrm{pH} 4.0$, supplemented with 7 Units of LOX-1, HPO lyase or $1 \mathrm{ml}$ of crude extract ( 1 Unit is the amount of enzyme that converts 1 $\mu \mathrm{mol} / \mathrm{min})$. Incubations with heat-denatured enzymes were performed as well. HHE and HNE were formed by incubating $25 \mu \mathrm{M}$ of (3Z)-hexenal or (3Z)-nonenal for $1 \mathrm{~h}$, in $2 \mathrm{ml}$ of $100 \mathrm{mM}$ potassium phosphate buffer pH 7.0, supplemented with $100 \mu \mathrm{M}$ 13-HPOD, 100 $\mu \mathrm{M}$ 13-HPOT, $100 \mu \mathrm{M} \mathrm{H}_{2} \mathrm{O}_{2}$, or with $100 \mu \mathrm{g}$ purified recombinant LOX-100. The reactions were stopped by reducing the hydroperoxy derivatives with an excess of $\mathrm{NaBH}_{4}$.

Analysis of 4-hydroxy-(2E)-alkenals. 9-Hydroxy-traumatin was extracted from the reaction mixture with an octadecyl solid-phase extraction column (J.T. Baker B.V., Deventer, The Netherlands) after acidification with $\mathrm{HCl}$ to $\mathrm{pH}$ 5.0, or with an Oasis HLB extraction cartridge (Waters, MA) without acidification, and eluted with $100 \%$ methanol. The product was reduced with an excess of $\mathrm{NaBH}_{4}$ at $0^{\circ} \mathrm{C}$, esterified with ethereal diazomethane and silylated with silylating reagent (pyridine/1,1,1,3,3,3-hexamethyldisilazane/chlorotrimethylsilane $5: 1: 1, v / v / v)$. Identification of the products was performed with GC/MS (Fisons GC 8000 series and Fisons I nstruments MD 800 MassLab spectrometer, Alltech AT-1 column, $0.25 \mu \mathrm{m}$ film thickness, $30 \mathrm{~m} \times 0.25 \mathrm{~mm}$ ), quantitative analysis was performed with GC/FID (Chrompack CP9002, CP-Sil5 CB column, $0.25 \mu \mathrm{m}$ film thickness, $25 \mathrm{~m} \times 0.32 \mathrm{~mm}$ ). The column temperature was held at $140^{\circ} \mathrm{C}$ for $2 \mathrm{~min}$, increased to $280^{\circ} \mathrm{C}, 6^{\circ} \mathrm{C} / \mathrm{min}$ and held at this temperature for $2 \mathrm{~min}$. Electron impact mass spectra were recorded with an ionization energy of $70 \mathrm{eV}$.

The analysis of HHE and HNE was performed as described before using some modifications (6). In order to form the 2,4dinitrophenylhydrazone (DNPH) derivatives of aldehydes, the incubation mixtures were stirred in the presence of $1 \mathrm{ml}$ of DNPHreagent $(0.1 \%$ 2,4-dinitrophenylhydrazine in $1 \mathrm{M} \mathrm{HCl})$ at room temperature for $1 \mathrm{~h}$. The reaction mixture was extracted three times with $5 \mathrm{ml}$ hexane each, and the collected organic phases were dried under a stream of $\mathrm{N}_{2}$. DNPH derivatives were redissolved in $400 \mu \mathrm{l}$ of acetonitrile. HPLC analysis of the DNPH derivatives was carried out by RP-HPLC on a J upiter C-18 300A column $(250 \times 1.0 \mathrm{~mm}, 5$ $\mu \mathrm{m}$ particle size, Phenomenex, Germany) using a binary gradient system (solvent A: acetonitrile/water $(60: 40, \mathrm{v} / \mathrm{v})$; solvent $B$ : acetonitrile/water $(80: 20, \mathrm{v} / \mathrm{v}))$ with the following gradient program: $100 \%$ solvent A for $15 \mathrm{~min}$, followed by a linear increase of solvent B up to $50 \%$ within $5 \mathrm{~min}$, a linear increase of solvent B up to $100 \%$ within $13.4 \mathrm{~min}$ and finally an isocratic postrun at $100 \%$ solvent $B$ for $11.6 \mathrm{~min}$. The flow rate was $0.05 \mathrm{ml} / \mathrm{min}$. The absorbance at $365 \mathrm{~nm}$ was monitored to indicate the presence of DNPH derivatives. All aldehydes were identified by their characteristic UV spectra and by co-injection of authentic standards. Calibration curves (five point measurements) were established.

\section{RESULTS}

To determine if the formation of 9-hydroxytraumatin was induced by the action of LOX, as proposed, traumatin was incubated with native or denatured LOX-1. Since formation of 9-hydroxy-traumatin was also observed in incubations of hydroperoxy fatty acids with HPO lyase $(10,11)$, and because many cytochrome P450 enzymes are known to have monooxygenase activity, it was also studied if the formation of 9-hydroxy-traumatin could be catalyzed by HPO lyase. Equal amounts were formed with active and denatured enzymes (Fig. 1A). The formation of hydroxytraumatin in these incubations is therefore due to autoxidation. With a crude extract of alfalfa a similar amount of hydroxy-traumatin was formed. Since the amount of hydroxy-traumatin formed in incubations at $\mathrm{pH} 6.0$ was higher than in incubations at $\mathrm{pH} 9.0$, the influence of the $\mathrm{pH}$ was studied by incubating traumatin in buffers with different $\mathrm{pH}$, with or without addition of LOX-1. The oxygenation of traumatin appeared to be higher at low pH (Fig. 1B) and was independent of the addition of LOX-1. When the sample of $\mathrm{pH} 9.0$ was not acidified prior to solid phase extraction, hardly any hydroxy-traumatin was formed. Interestingly, a novel product derived from traumatin was observed. This product was identified as 12-oxo-11-hydroxy-(9Z)dodecenoic acid (11-hydroxy-traumatin) (Fig. 2). The amount was about 5 times less than the amount of 9-hydroxy traumatin formed.

An incubation in $\mathrm{H}_{2}{ }^{18} \mathrm{O}$ (Fluka) was carried out to determine if the oxygen from the hydroxy group is derived from water or from molecular oxygen. The mass spectra of the formed 9- and 11-hydroxytraumatin only demonstrated exchange of oxygen of the $\mathrm{C}_{12}$-aldehyde group but no incorporation of ${ }^{18} \mathrm{O}$ in the hydroxy group at either $\mathrm{C}_{9}$ or $\mathrm{C}_{11}$. It is, therefore, likely that 9- and 11-hydroxy-traumatin are formed by a reaction of 12-oxo-(9Z)-dodecenoic acid with molecular oxygen.

To determine if $\mathrm{HHE}$ and HNE were formed in an enzymatic reaction, (3Z)-hexenal or (3Z)-nonenal were incubated with recombinant LOX-100, which should be the enzyme responsible for $\mathrm{HHE}$ formation in case of barley leaves (6). Furthermore, (3Z)-hexenal or (3Z)nonenal were incubated with oxidizing chemicals such as 13-HPOD, 13-HPOT or $\mathrm{H}_{2} \mathrm{O}_{2}$, or with a combination of 13-HPOD/T or $\mathrm{H}_{2} \mathrm{O}_{2}$ and LOX-100. For both aldehydes similar results were obtained. As shown for HNE formation (Fig. 3), similar values were found when (3Z)-nonenal was incubated with 13-HPOD/T only, or in combination with LOX-100. These results 
A

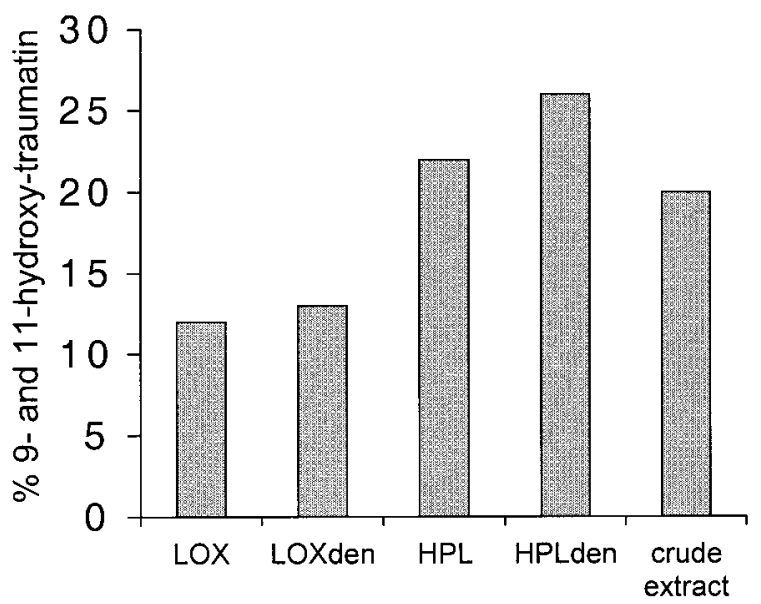

B

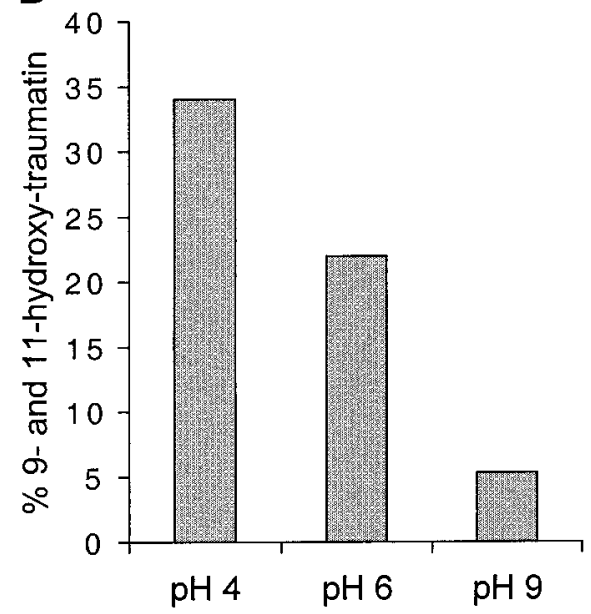

FIG. 1. Formation of 9- and 11-hydroxy traumatin as a percentage of the total amount of 12-oxo-dodecenoic acids present. (A) Formation of 9- and 11-hydroxy traumatin by native or denatured LOX-1 ( $\mathrm{pH} \mathrm{9.0),} \mathrm{HPO} \mathrm{lyase} \mathrm{(pH} \mathrm{6.0)} \mathrm{or} \mathrm{a} \mathrm{crude} \mathrm{extract} \mathrm{of} \mathrm{alfalfa} \mathrm{(} \mathrm{pH} 6.0$ ). (B) Formation of 9- and 11-hydroxy-traumatin in buffers of different pH. Quantification occurred with GC/FID after extraction and derivatization of the products. Representative results are shown.

indicate that the formation of HHE and HNE under these conditions is due to autoxidation as well. The formation of 9- and 11-hydroxy-traumatin did not increase in the presence of $100 \mu \mathrm{M}$ 13-HPOD, which is in contrast to the formation of HHE and HNE. Furthermore, incubation of 12-oxo-(9Z)-dodecenoic acid with $100 \mu \mathrm{M} 13-\mathrm{HP} \mathrm{P}^{18} \mathrm{OD}$ did not result in incorporation of ${ }^{18} \mathrm{O}$.
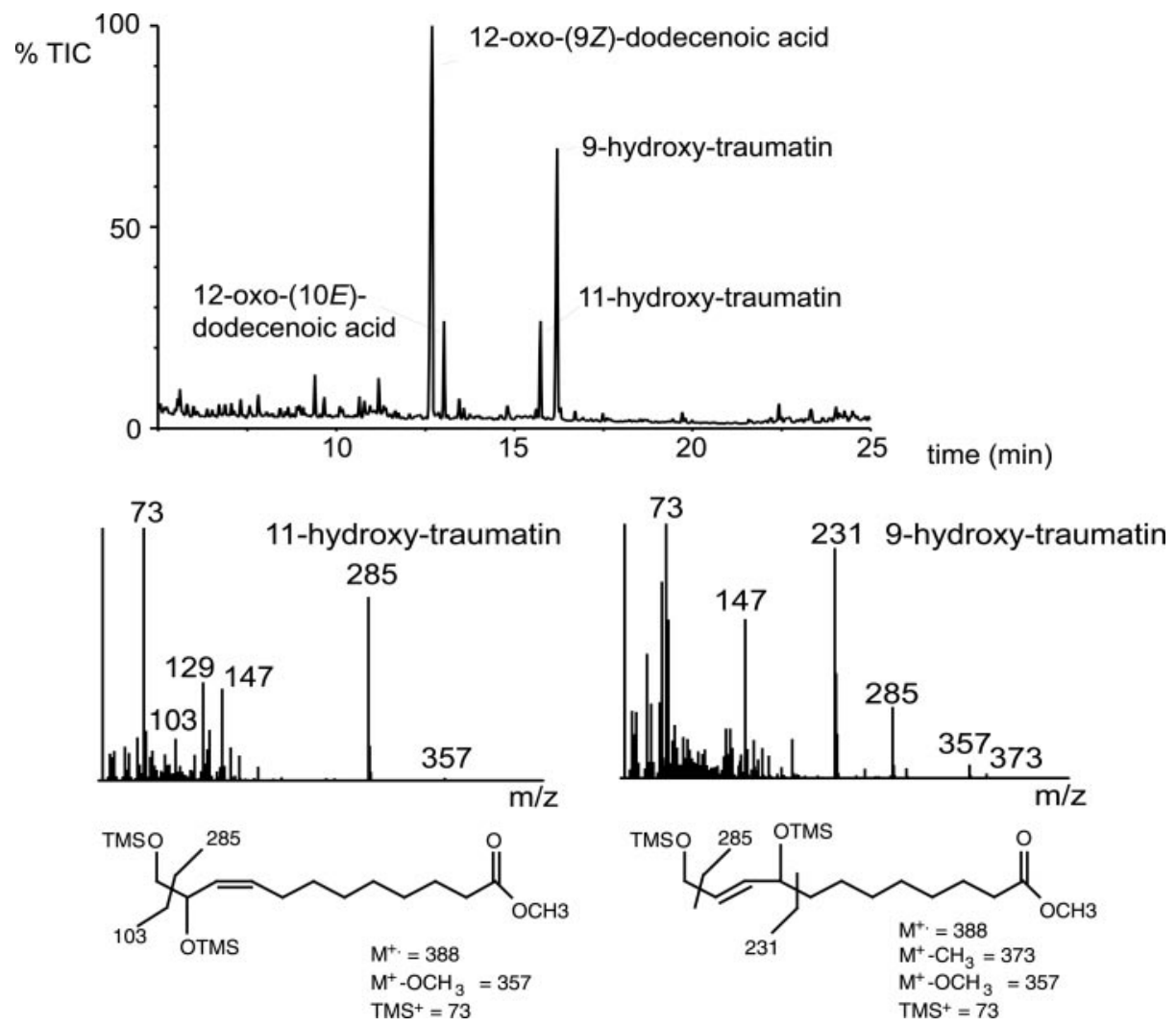

FIG. 2. Formation of 9- and 11-hydroxy-traumatin upon incubation of 12-oxo-(9Z)-dodecenoic acid in $50 \mathrm{mM}$ sodium acetate buffer pH 4.0 for $1.5 \mathrm{~h}$. The products were extracted, derivatized, and identified with GC/MS. 


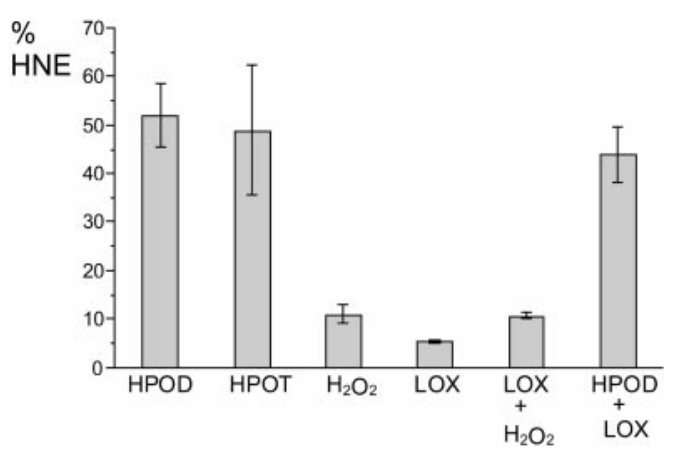

FIG. 3. Formation of HNE upon incubation of (3Z)-nonenal with $100 \mu \mathrm{M}$ 13-HPOD, $100 \mu \mathrm{M}$ 13-HPOT, $100 \mu \mathrm{M} \mathrm{H}_{2} \mathrm{O}_{2}$, or with $100 \mu \mathrm{g}$ purified recombinant LOX 100, respectively, for $1 \mathrm{~h}$. The remaining aldehydes were derivatized and analyzed by HPLC. The results represent the mean and the standard deviation of 3 experiments.

\section{DISCUSSION}

In contrast to the previously proposed enzymatic routes for the formation of 4-hydroxy-(2E)-alkenals in plants, we have shown here that $\mathrm{HHE}, \mathrm{HNE}$ and 9-hydroxy-traumatin can be formed in a nonenzymatic process. Until now, it had not been studied if 9-hydroxy-traumatin found in crude extracts, could be due to autoxidation. The previously described formation of HNE by LOX-1 spiked with 13-HPOD (9) might be mainly due to the presence of 13-HPOD and not to the action of LOX-1, since we showed that 13-HPOD and 13-HPOT alone induced the formation of much higher amounts of HHE and HNE than LOX. An autoxidative process may also explain the inability of LOX inhibitors to inhibit the oxygenation of (3Z)alkenals $(8,15)$ and the almost racemic mixture of products obtained with crude enzyme preparations (8, 9). The fact that there was no formation of HNE observed with heat-inactivated enzyme preparations in those previous studies, might be due to the reaction conditions used. Since (3Z)-nonenal was found to disappear in the incubations with denatured enzyme as well $(8,15)$, it might be possible that the formed HNE was not recovered because of the formation of Schiff bases, as was also proposed for 9-hydroxy-traumatin in undiluted enzyme preparations (5). In addition, it has been suggested that formation of 4-hydroxy-(2E)alkenals is catalyzed by 9-LOX activity. However, even at neutral pH where LOX-1 has significant 9-LOX activity (17), we could not find enzymatic formation of these oxylipins.

We also studied the reaction itself in more detail. Incubations with $\mathrm{H}_{2}{ }^{18} \mathrm{O}$ showed that ${ }^{18} \mathrm{O}$ was not incorporated into 9-hydroxy-traumatin, indicating that the hydroxy group is not derived from water. Previously, $\mathrm{O}_{2}$-uptake during the formation of hydroxy alkenals and the formation of hydroperoxy alkenals was observed $(9,15)$. This indicates that the oxygenation of
(3Z)-alkenals to 4-hydroxy-(2E)-alkenals is a reaction with molecular oxygen. Furthermore, we observed no incorporation of ${ }^{18} \mathrm{O}$ in 9- and 11-hydroxy traumatin upon incubation of traumatin with $13-H P^{18} \mathrm{OD}$. Gardner and Hamberg found some labelled HNE upon incubation of (3Z)-nonenal with $13-\mathrm{HP}^{18} \mathrm{OD}$, but only to an extent of $15.7 \%$ (8). This suggests that the majority of 4-hydroxy-(2E)-alkenals is not formed by a direct interaction with 13-HPOD/T. The induced formation of $\mathrm{HHE}$ and HNE by 13-HPOD/T might therefore be due to initiation of the oxygenation by radicals generated by $13-\mathrm{HPOD} / \mathrm{T}$. The formation of both 9- and 11hydroxy-traumatin indicates that the reaction might occur via a $\mathrm{C}_{11}-\mathrm{C}_{9}$ del ocalized radical intermediate. The lower amount of 11-hydroxy-traumatin compared to the amount of 9-hydroxy-traumatin formed, might be due to the proximity of the oxygen of the aldehyde group, which could make an oxygen attack at $\mathrm{C}_{11}$ less favorable than at $\mathrm{C}_{9}$. The influence of the $\mathrm{pH}$ on the oxygenation remains unclear, since formation of 9- and 11-hydroxy-traumatin was optimal at low $\mathrm{pH}$, whereas the formation of HNE and HHE was optimal at $\mathrm{pH} 9.5$ (15).

The results obtained here show that HHE, HNE and 9-hydroxy traumatin, are in vitro formed by autoxidation of (3Z)-hexenal, (3Z)-nonenal and 12-oxo-(9Z)dodecenoic acid, probably similar to the autoxidation of linoleic and linolenic acid. The 400- to 1000-fold lower activity of LOX-1 with (3Z)-nonenal compared to linoleic acid (9), questions the significance of this enzymatic reaction in vivo. It is therefore likely that the previously observed formation of 4-hydroxy-(2E)alkenals in crude extracts of plants is mainly due to autoxidation. This might have implications for the previously proposed physiological significance of these products in plants. However, 4-hydroxy-(2E)-alkenals could be excellent markers to distinguish between autoxidative and enzymatic processes.

\section{ACKNOWLEDGMENTS}

This work was financially supported in part by a grant to I.F. by the Deutsche Forschungsgemeinschaft (Fe 446/1-2). The authors thank Professor Dr. C. Wasternack, Halle, for critical reading the manuscript.

\section{REFERENCES}

1. Benedetti, A., Comporti, M., and Esterbauer, H. (1980) Identification of 4-hydroxynonenal as a cytotoxic product originating from the peroxidation of liver microsomal lipids. Biochim. Biophys. Acta 620, 281-296.

2. Esterbauer, H., Schauer, R. J ., and Zollner, H. (1991) Chemistry and biochemistry of 4-hydroxynonenal, malonaldehyde and related aldehydes. Free Radic. Biol. Med. 11, 81-128.

3. Pryor, W. A., and Porter, N. A. (1990) Suggested mechanisms for the production of 4-hydroxy-2-nonenal from the autoxidation of polyunsaturated fatty acids. Free Radic. Biol. Med. 8, 541-543.

4. Gardner, H. W., Weisleder, D., and Plattner, R. D. (1991) Hy- 
droperoxide lyase and other hydroperoxide-metabolizing activity in tissues of soybean, Glycine-max. Plant Physiol. 97, 10591072.

5. Gardner, H. W. (1998) 9-hydroxy-traumatin, a new metabolite of the lipoxygenase pathway. Lipids 33, 745-749.

6. Kohlmann, M., Bachmann, A., Weichert, H., Kolbe, A., Balkenhohl, T., Wasternack, C., and Feussner, I. (1999) Formation of lipoxygenase-pathway-derived aldehydes in barley leaves upon methyl jasmonate treatment. Eur. J . Biochem. 260, 885- 895.

7. Noordermeer, M. A., Veldink, G. A., and Vliegenthart, J. F. G. (1999) Alfalfa contains substantial 9-hydroperoxide lyase activity and a 3Z:2E-enal isomerase. FEBS Lett. 443, 201-204.

8. Gardner, H. W., and Hamberg, M. (1993) Oxygenation of (3Z)nonenal to (2E)-4-hydroxy-2-nonenal in the broad bean (Vicia faba L.). J . Biol. Chem. 268, 6971-6977.

9. Gardner, H. W., and Grove, M. J . (1998) Soybean lipoxygenase-1 oxidizes 3Z-nonenal. Plant Physiol. 116, 1359-1366.

10. Noordermeer, M. A., Dijken, A. J. H. v., Smeekens, S. C. M., Veldink, G. A., and Vliegenthart, J. F. G. (2000) Characterization of three cloned and expressed 13-hydroperoxide lyase isoenzymes from alfalfa with unusual $\mathrm{N}$-terminal sequences and different enzyme kinetics. Eur. J . Biochem. 267, 2473-2482.

11. Stelt, M. v. d., Noordermeer, M. A., Kiss, T., Zadelhoff, G. v., Merghart, B., Veldink, G. A., and Vliegenthart, J. F. G. (2000)
Formation of a new class of oxylipins from $\mathrm{N}$-acyl(ethanol)amines by the lipoxygenase pathway. Eur. J . Biochem. 267, 2000-2007.

12. Finazzi-Agrò, A., Avigliano, L., Veldink, G. A., Vliegenthart, J . F. G., and Boldingh, J . (1973) The influence of oxygen on the fluorescence of lipoxygenase. Biochim. Biophys. Acta 326, 462470.

13. Vörös, K., Feussner, I., Kühn, H., Lee, J ., Graner, A., Löbler, M., Parthier, B., and Wasternack, C. (1998) Characterization of a methyljasmonate-inducible lipoxygenase from barley (Hordeum vulgare cv. Salome) leaves. Eur. J . Biochem. 251, 36- 44.

14. Feussner, I., Hause, B., Vörös, K., Parthier, B., and Wasternack, C. (1995) J asmonate-induced lipoxygenase forms are localized in chloroplasts of barley leaves (Hordeum vulgare cv. Salome). Plant J. 7, 949-957.

15. Takamura, H., and Gardner, H. W. (1996) Oxygenation of (3Z)alkenal to (2E)-4-hydroxy-2-alkenal in soybean seed (Glycine max L). Biochim. Biophys. Acta 1303, 83-91.

16. Elshof, M. B. W., J anssen, M., Veldink, G. A., and Vliegenthart, J. F. G. (1996) Biocatalytic large-scale production of 13(S)hydroperoxy-(9Z,11E)-octadeca-9,11-dienoic acid from hydrolysed safflower oil by a crude soybean-flour extract as lipoxygenase source. Rec. Trav. Chim. Pays-Bas 115, 499-504.

17. Gardner, H. W. (1989) Soybean lipoxygenase-1 enzymatically forms both (9S)- and (13S)-hydroperoxides from linoleic acid by a pH-dependent mechanism. Biochim. Biophys. Acta 1001, 274281. 